\title{
Diretivas antecipadas de vontade na assistência hospitalar: perspectiva de enfermeiros
}

Silvana Bastos Cogo ${ }^{1}$, Elisabeta Albertina Nietsche ${ }^{1}$, Marcio Rossato Badke ${ }^{1}$, Graciela Dutra Sehnem ${ }^{1}$, Cléton Salbego ${ }^{1}$, Tierle Kosloski Ramos ${ }^{1}$, Andrei Pompeu Antunes ${ }^{1}$, Aline Gomes Ilha ${ }^{1}$, Luiza Carolina Santos Malheiros ${ }^{1}$

1. Universidade Federal de Santa Maria, Santa Maria/RS, Brasil.

\begin{abstract}
Resumo
O objetivo deste estudo é compreender como enfermeiros assistenciais de hospital universitário atuariam em casos de pacientes em fase final de vida providos de diretivas antecipadas de vontade. Trata-se de estudo qualitativo, descritivo e exploratório realizado com 20 enfermeiros de um hospital universitário e público do Sul do Brasil. Os dados foram coletados de novembro de 2017 a janeiro de 2018, por meio de entrevistas individuais semiestruturadas, e submetidos a análise textual discursiva, a partir da qual foram elencadas três categorias: aceitação da morte no contexto hospitalar como pressuposto para cumprir as diretivas; autonomia do paciente, respeitando limites; e diretiva antecipada de vontade, do (des)conhecimento à possibilidade de respaldo profissional. Concluiu-se que os enfermeiros consideram que as diretivas antecipadas de vontade facilitam o respeito à autonomia do paciente, além de oferecerem respaldo ao profissional.
\end{abstract}

Palavras-chave: Enfermeiras e enfermeiros. Diretivas antecipadas. Assistência hospitalar. Ética em enfermagem. Autonomia pessoal.

\section{Resumen}

Directivas anticipadas de voluntad en la atención hospitalaria: perspectiva de enfermeros

El objetivo de este estudio es comprender cómo actuarían los enfermeros asistenciales de hospitales universitarios en casos de pacientes en la fase final de la vida provistos de directivas anticipadas de voluntad. Se trata de un estudio cualitativo, descriptivo y exploratorio con 20 enfermeros de un hospital universitario y público del Sur de Brasil. Los datos fueron recolectados de noviembre de 2017 a enero de 2018, a través de entrevistas semiestructuradas individuales. A partir de un análisis textual discursivo, los datos se clasificaron en tres categorías: aceptación de la muerte en el contexto hospitalario como prerrequisito para el cumplimiento de las directivas; autonomía del paciente, respetando límites; y directiva anticipada de voluntad, desde el (no) conocimiento hasta la posibilidad de apoyo profesional. Se concluyó que los enfermeros consideran que las directivas anticipadas de voluntad facilitan el respeto a la autonomía del paciente, además de ofrecer apoyo al profesional.

Palabras clave: Enfermeras y enfermeros. Directivas anticipadas. Atención hospitalaria. Ética en enfermería. Autonomía personal.

\section{Abstract}

\section{Advance healthcare directives in hospital care: nurses' perspective}

This study aims to understand how nursing assistants from a university hospital would act in cases of end-of-life patients provided with advance directives. This is a qualitative, descriptive and exploratory study conducted with 20 nurses from a public university hospital in the South of Brazil. Data were collected from November 2017 to January 2018, using semi-structured individual interviews, and submitted to discursive textual analysis, from which we established three categories: acceptance of death in the hospital context as prerequisite for complying with the directives; patient autonomy, respecting limits; advance healthcare directives: from (lack of) knowledge to the possibility of professional support. In conclusion, nurses consider that advance healthcare directives facilitate fulfilling patient autonomy, besides offering support to the nursing professional.

Keywords: Nurses. Advance directives. Hospital care. Ethics, nursing. Personal autonomy. 
O envelhecimento populacional vem resultando na sobrecarga dos serviços de saúde, tendo em vista que essa fase do ciclo vital é caracterizada pelo acúmulo de danos moleculares e celulares, além de doenças crônicas que podem surgir ao longo dos anos. Esse aumento da expectativa de vida associa-se à evolução da tecnologia, bem como ao tratamento modificador da doença, especialmente daquelas em estágio progressivo e avançado, que ameaçam a vida ${ }^{1}$.

Essa tendência vem acompanhada de obstinação terapêutica - o intuito de afastar a morte e prolongar a vida mesmo que sem condições dignas. Tal prática, que resulta em morte lenta e dolorosa, pode ser considerada degradante e desumana ${ }^{2}$. Por isso, atualmente o Brasil segue a predisposição mundial de afirmar o direito à autonomia, que visa permitir à pessoa escolher a conduta que prefere em situações incertas e delicadas ${ }^{3}$. A maioria dos pacientes gravemente enfermos, no entanto, perde a capacidade de tomar decisões complexas; nesses casos, as resoluções tomam como base critérios da família e do médico assistente ${ }^{4}$.

Visando resguardar a autonomia do paciente nessas condições, surgiram as diretivas antecipadas de vontade (DAV), que abarcam documentos como o testamento vital e o mandato duradouro ${ }^{5}$. De acordo com Dadalto ${ }^{6}$, as DAV foram propostas pela primeira vez em 1969 em texto de Luis Kutner, advogado estadunidense defensor dos direitos humanos. Ao publicar o primeiro artigo sobre o tema, Kutner criou documento nomeado "living will", que continha as premissas do testamento vital. Em 1991, o documento se tornou lei nos Estados Unidos com o Patient Self-Determination Act (PSDA), que em sua segunda seção dispõe sobre as Advanced Healthcare Directives, conhecidas no Brasil como "diretivas antecipadas de vontade" ${ }^{6}$. Após a PSDA americana, a Europa começou a legalizar as DAV na década de 1990; na América Latina, somente a Argentina e o Uruguai as regulamentaram, em $2009^{6}$.

É importante destacar que o testamento vital e as DAV são institutos distintos, embora muitos estudos se equivoquem ao tratá-los como sinôni$\operatorname{mos}^{4}$. As DAV possibilitam ao paciente exercer o direito à liberdade e à autonomia ${ }^{7}$ e visam garantir o respeito à dignidade humana ${ }^{5}$. Elas podem ser registradas em testamento vital e/ou mandato duradouro. O testamento vital é redigido por pessoa em pleno gozo de suas faculdades mentais, prevendo situação em que não possa manifestar livremente sua vontade. O objetivo é dispor acerca de cuidados, tratamentos e procedimentos - como ressuscitação cardiopulmonar, ventilação mecânica, dieta artificial, medicações, fluídos intravenosos etc. - a que deseja ou não ser submetida diante de doença ameaçadora da vida, em estágio progressivo e avançado, fora de possibilidade terapêutica de cura ${ }^{4}$. Já o mandato duradouro tem por objetivo nomear um procurador de saúde, que será responsável por decidir em nome do paciente quando este não o puder fazer ${ }^{6}$.

Apesar de sua importância e recorrência na atividade médica diária, em geral os profissionais de saúde brasileiros têm pouca familiaridade com as DAV $^{3}$. Em 2012, o Conselho Federal de Medicina ${ }^{8}$ promulgou a Resolução 1.995 , que dispõe sobre a aplicação das DAV, definindo-as como o conjunto de desejos prévia e expressamente manifestados pelo paciente sobre cuidados e tratamentos que quer ou não receber caso esteja incapacitado de expressar livre e autonomamente sua vontade. Dessa maneira, a resolução preserva a autonomia do paciente, que pode recusar tratamentos ou o prolongamento de sua vida por meios artificiais ${ }^{7}$. As DAV, no entanto, não têm respaldo legal - o que não quer dizer que sejam ilícitas.

Em outros países, a prática já vigora há décadas, e constata-se que, embora ainda haja dificuldades quanto à sua aplicação, as DAV são bem aceitas pela população e por pacientes e profissionais da saúde ${ }^{9-11}$. A importância do instrumento foi evidenciada por análise da produção científica sobre DAV de pacientes acometidos por doença terminal, que mostrou ainda a viabilidade do dispositivo quando devidamente inserido na relação paciente-familiar-profissional, considerando o respeito à autonomia e à dignidade humana ${ }^{12}$.

Nesse contexto, o profissional enfermeiro se destaca, visto que permanece por mais tempo junto ao paciente e seus familiares, interagindo ainda com toda a equipe multiprofissional. Isso viabiliza comunicação qualificada, tanto para orientar como para provocar reflexões e discussões sobre as DAV ${ }^{13}$.

O Código de Ética dos Profissionais de Enfermagem ${ }^{14}$ menciona as diretivas antecipadas e estabelece que devem ser respeitadas, mas o enfermeiro que se sentir inseguro com a ausência de legislação específica acerca do tema encontra respaldo no seu artigo 22 , que descreve o direito 
de se recusar a executar atividades que não ofereçam segurança ao profissional. A falta de amparo legal gera incerteza na aplicação do dispositivo. Soma-se a isso, ainda, a abordagem deficitária do tema da terminalidade na formação acadêmica desses profissionais, que acaba por esvaziar a função das diretivas, desconsiderando sua relevância no direcionamento das condutas terapêuticas ${ }^{15}$.

Diante do exposto, considera-se que a aplicação das DAV pode apoiar a enfermagem em dilemas e conflitos éticos ${ }^{12}$ - especialmente quando as condutas médicas não estão explicitadas, como nas ordens de não reanimar. Dessa forma, este estudo se justifica pela relevância de seu objetivo: conhecer a perspectiva de enfermeiros de um hospital universitário acerca da possibilidade de atender paciente que registrou diretivas antecipadas, tentando compreender como atuariam nessa situação. Mais especificamente, questionou-se aos enfermeiros como compreendiam sua atuação diante de pacientes em fim de vida providos de DAV.

\section{Método}

Trata-se de estudo qualitativo, descritivo e exploratório, desenvolvido em duas unidades de clínica médica (CM I e CM II), uma clínica cirúrgica (CC) e uma unidade de terapia intensiva (UTI) adulto de hospital universitário e público de grande porte do Sul do Brasil. No momento da pesquisa, a CM I contava com 10 enfermeiros; a CM II, com 11; a clínica cirúrgica, com 17; e a UTI, com $11^{16}$. A CM I atende pacientes com patologias relacionadas à nefrologia ou à oncologia; na CM II estão os pacientes internados devido a complicações de doenças crônico-degenerativas; na clínica cirúrgica, pacientes que realizarão procedimentos cirúrgicos; e, por fim, na UTI encontram-se os pacientes em estado grave, que necessitam de cuidados intensivos ${ }^{16}$.

A seleção dos enfermeiros foi intencional, conforme os critérios de inclusão: ser enfermeiro da CM I, CM II, CC ou UTI e atuar há pelo menos um ano na unidade (período mínimo que, presume-se, possibilitaria aos enfermeiros contar com experiências mais abrangentes). Além dos profissionais que não atendessem aos critérios de inclusão, foram excluídos aqueles que estavam em licença médica ou afastados do trabalho. A inclusão de participantes na amostra e a coleta de dados foram encerradas por saturação - isto é, quando as informações começaram a se repetir e se pôde alcançar os objetivos propostos, não se realizaram novas entrevistas. Dos 22 profissionais convidados antes do fechamento da amostra, dois não aceitaram participar, afirmando desconhecer o tema. Antes da coleta de dados, realizou-se teste-piloto para avaliar o roteiro de entrevista, que se mostrou adequado.

Os dados foram coletados de novembro de 2017 a janeiro de 2018, e inicialmente foi aplicado instrumento elaborado pelos autores para colher dados sociodemográficos dos participantes. Depois isso, disponibilizou-se aos enfermeiros modelo de DAV e, a partir desse documento, iniciou-se a entrevista semiestruturada individual. As questões norteadoras abrangeram: conhecimento sobre as DAV; experiências com as DAV no contexto hospitalar; atuação diante de paciente que se recusa a ser submetido a procedimentos e tratamentos considerados extraordinários; cumprimento pela equipe de enfermagem das vontades manifestadas pelo doente; conduta em casos de conflito entre a decisão do paciente, dos familiares e dos profissionais da saúde; e importância da enfermagem no contexto das DAV.

As entrevistas foram conduzidas individualmente por uma das pesquisadoras e em sua maioria ocorreram no ambiente de trabalho dos enfermeiros, em salas privativas, a fim de manter a privacidade e o sigilo, conforme conveniência e disponibilidade, sem interferir no andamento das atividades assistenciais. As entrevistas foram gravadas para transcrição posterior, totalizando 610 minutos de áudio, com duração média de aproximadamente 30 minutos por entrevista.

As falas foram transcritas pela pesquisadora que coletou os dados. As informações foram organizadas no software Microsoft Excel e submetidas a análise textual discursiva, que visa desconstruir e reconstruir a compreensão do pesquisador a fim de que novos entendimentos emerjam dos fenômenos investigados. Tal análise percorre três etapas: 1) unitarização, que consiste na desconstrução dos textos do corpus; 2) categorização, que estabelece relações entre os elementos unitários; e 3) captação do novo, em que compreensão emergente é comunicada e validada ${ }^{17}$.

$\mathrm{Na}$ fase de unitarização, as entrevistas foram examinadas detalhadamente a fim de que se pudessem identificar unidades constituintes do fenômeno 
estudado ${ }^{17}$. Essa etapa, por sua vez, também seguiu três passos: 1) fragmentação dos textos e codificação de cada unidade; 2) reescrita de cada unidade de modo a reconhecer nela um significado; e 3) atribuição de um título para cada unidade.

$\mathrm{Na}$ etapa seguinte, de categorização, as unidades de análise foram organizadas e agrupadas a fim de compor categorias que estabelecessem relações entre os elementos ${ }^{17}$. Já na terceira etapa, relacionada a nova compreensão do fenômeno estudado, produziram-se metatextos (de teor descritivo e interpretativo) a partir das unidades de análise e das categorias. Essa produção possibilitou a emergência de um entendimento renovado dos dados coletados ${ }^{17}$.

A pesquisa respeitou os preceitos éticos estabelecidos na Resolução 466/2012 do Conselho Nacional de Saúde ${ }^{18}$. Os participantes foram identificados com códigos alfanuméricos formados pela sigla "EP", de "enfermeiro participante", seguida do número de ordem das entrevistas.

\section{Resultados e discussão}

Participaram da pesquisa 20 enfermeiros 16 mulheres e 4 homens -, abrangendo as faixas etárias de 20 a 30 anos (7 participantes), 30 a 40 anos (12 participantes) e mais de 40 anos (1 participante). Entre as unidades pesquisadas, a CC teve mais participantes, com 6 enfermeiros, seguida pela UTI e CM II, com 5 participantes cada, e a CM I, com 4 participantes. Em relação ao tempo de experiência profissional no hospital pesquisado, 11 participantes atuam como enfermeiros há entre 5 e 10 anos; 3 há mais de 10 anos; e 6 há menos de cinco anos. Da análise dos dados emergiram três categorias que representam como os enfermeiros atuariam, hipoteticamente, ao cuidar de pacientes em fase final de vida providos de DAV.

\section{Aceitação da morte no contexto hospitalar: pressuposto para cumprir diretivas antecipadas}

Quanto à aplicação das DAV, as condutas e reações dos enfermeiros passam pela questão da finitude, já que todos os enfermeiros entrevistados relataram a experiência de acompanhar a morte de algum paciente. Foi possível perceber nas entrevistas que a morte e as diretivas antecipadas são aceitas com mais naturalidade quando o paciente apresenta doença crônica avançada:

"A terminalidade, ela existe do PS [pronto-socorro] ao sexto andar [pediatria], inclusive para mim e pra ti. Mas a ideia de fazer o que o paciente deixou escrito, parece que é mais fácil quando acontece com o paciente que é mais velho, que já tem uma doença de bastante tempo e que está sofrendo" (EP2).

Pode-se inferir que quando a equipe aceita a morte há maior consenso quanto à aplicação das DAV, já que, se o paciente apresenta prognóstico negativo, seguir tais diretivas parece mais aceitável aos profissionais. Sobre esse aspecto, pesquisa com enfermeiros constatou que a morte de pacientes idosos ou com doenças consideradas terminais foram mais facilmente aceitas pelos profissionais, visto que nesses casos o óbito foi visto como o desfecho esperado ${ }^{19}$. Tal atitude, porém, pode ser vista como forma de se proteger contra o inevitável, uma vez que ela se modifica quando a equipe sente a morte do paciente como quebra no ciclo natural de vida ${ }^{19}$.

Essa conclusão é corroborada neste estudo, pois, mesmo que convivam cotidianamente com a terminalidade dos doentes, é difícil para os enfermeiros encarar a morte de pacientes jovens, o que pode prejudicar a aplicação das DAV:

"É difícil lidar com a morte de pacientes jovens. Como [é mais comum] a gente enfrenta[r] a morte de um paciente crônico, idoso, que está exercendo o estágio da doença [em processo de final de vida], [quando] um paciente [...] sofre um politrauma, que é jovem, a gente fica extremamente abalado com isso, porque a gente é ser humano. (...) Nestes casos [de pacientes jovens], já seria mais difícil cumprir as diretivas" (EP11).

"Se é um paciente idoso com uma doença terminal, que já estava com a qualidade de vida debilitada, parece que é mais fácil a aceitação de respeitar o que eles deixaram escrito, do que gostariam que fosse feito para eles. Se é um paciente jovem, que não tem o diagnóstico bem esclarecido e vem a óbito, aí a gente acaba sofrendo um pouco mais e correria o risco de não respeitar [as DAV], pois não sabemos o que de fato irá acontecer" (EP12). 


\section{Autonomia do paciente: respeitando limites}

A aplicação das DAV é apontada pelos enfermeiros como forma de respeitar a autonomia do paciente em final de vida:

"O paciente deve ter autonomia para escolher o tipo de tratamento, no caso de uma patologia grave, no caso de um paciente terminal, por exemplo, se vai ser feita uma cirurgia ou não, como acontece em alguns casos aqui" (EP8).

"A gente tem que respeitar, porque é a vontade dele. Teve situações em que os pacientes não quiseram fazer tratamento; ninguém é obrigado a fazer. Se tem essa vontade expressa, eu acho que ela tem que ser respeitada" (EP15).

$\mathrm{Na}$ complexa relação entre paciente e equipe de saúde, é necessário encontrar equilíbrio entre o poder individual e os demais poderes. Dessa maneira, quanto melhor a relação, melhores serão as decisões tomadas para não causar dano ao paciente, respeitando sua autonomia em todos os momentos ${ }^{20}$. Nesse sentido, os profissionais destacaram que a vontade expressa nas diretivas antecipadas pode ser garantida se as determinações estiverem em conformidade com as rotinas da unidade e da instituição:

"A partir do momento em que um paciente estiver com o instrumento [DAV] em mãos, da minha parte, eu iria respeitar ao máximo, dentro das rotinas da unidade. Mas a gente sempre faz com que prevaleça a vontade do paciente" (EP3).

Em pesquisa realizada em outro hospital universitário do Sul do país, os profissionais abordados também destacaram a importância de respeitar a autonomia dos pacientes, mas disseram se sentir inseguros em seguir as DAV por não haver legislação que os resguarde ${ }^{15}$. Outro estudo revelou que a maior parte dos entrevistados (enfermeiros, médicos e farmacêuticos) disse que respeitaria a vontade expressa em DAV se estas fossem juridicamente vinculativas ${ }^{21}$.

Nesse sentido, a falta de amparo legal e a abordagem deficitária da terminalidade durante a formação acadêmica - tradicionalmente concentrada na técnica e na cura - geram insegurança e limitam a função das DAV, tratadas como mera informação adicional, sem grande relevância para direcionar as condutas terapêuticas ${ }^{15}$. Embora reconheçam a importância de saber a vontade do enfermo, os profissionais sentem a necessidade de se resguardar de possíveis problemas judiciais.

Também foram identificadas afirmativas que ressaltam o direito do paciente de escolher que tratamentos e cuidados deseja ou não receber. Essa autonomia precisa de fato ser compreendida pelo enfermeiro, a fim de que possa respeitar as decisões expressas mesmo que não correspondam aos desejos do profissional:

"Eu vejo que tem profissionais que querem discutir com o paciente, que tem que fazer e pronto. Mas não é assim que funciona. Você tem o direito de escolha sobre teu corpo, teu cuidado, teu tratamento; a gente tem que aprender a respeitar isso, mesmo a gente não concordando com o documento [DAV] que ele escreveu" (EP13).

Estudo já citado, com enfermeiros, médicos e farmacêuticos, mostrou que a maioria dos entrevistados considerava que debater decisões de final de vida com o enfermo é ação ética e aceitável, levando a interpretar que os profissionais de saúde tendem a respeitar a autonomia do paciente e a apoiar a morte digna ${ }^{21}$. Na presente pesquisa, os depoimentos indicam que a autonomia é considerada importante e deve ser respeitada, ainda que as manifestações do paciente não sejam reconhecidas como as mais adequadas na perspectiva pessoal dos profissionais.

Há de se destacar, no entanto, que a terminalidade é marcada por dúvidas, angústias, receios e medos. É importante que os profissionais reconheçam e aceitem a morte, comunicando essa situação a pacientes e familiares de modo a promover mudanças nas atitudes diante da finitude. O desejo de respeitar as vontades do paciente não é suficiente se o profissional é incapaz de aceitar a morte. A sensação de impotência dos profissionais limita a implementação das DAV e favorece a obstinação terapêutica ${ }^{22}$.

\section{Diretivas antecipadas de vontade: do (des) conhecimento à possibilidade de respaldo profissional}

Como mostram as falas dos entrevistados, alguns profissionais desconhecem as DAV, atribuindo a lacuna à falta de aproximação com o tema durante a graduação. Entretanto, os participantes 
ressalvam que, embora não utilizem a nomenclatura, as escolhas manifestadas pelos pacientes são respeitadas no hospital:

"Na faculdade a gente não tem conhecimento disso, não é falado, e nem aqui no hospital. Eu entendi sobre o que se trata, mas até então eu desconhecia o que poderia ser feito e tudo mais, pelo próprio paciente, ou até de ter uma pessoa responsável, porque a gente se depara com essas situações, mas nunca foram usados esses termos das diretivas" (EP5).

O resultado corrobora pesquisa realizada no distrito de Kaunas, na Lituânia, que constatou baixo nível de conhecimento sobre DAV: apenas $16,7 \%$ dos entrevistados afirmaram conhecer o significado das "diretivas antecipadas" - dentre eles, médicos estavam mais familiarizados com o termo do que enfermeiros e farmacêuticos ${ }^{21}$. No presente estudo, o desconhecimento parece estar relacionado a limitações na formação acadêmica, que, voltada à preservação e permanência da vida, não trabalha adequadamente a finitude, tratando-a como insucesso. Além disso, as experiências da graduação nem sempre são suficientes para preparar o profissional para lidar com as DAV e atuar com pacientes e familiares que vivenciam o processo de morte ${ }^{23,24}$.

Pesquisa com estudantes de enfermagem constatou maior aceitação das DAV à medida que os semestres avançavam, havendo progressão significante do conhecimento e das atitudes pessoais em relação a esse dispositivo ${ }^{24}$. O resultado mostra como é importante que os futuros profissionais sejam expostos ao tema. Afinal, trata-se de instrumento que, como apontam os entrevistados, promove o respeito às vontades previamente expressas pelo paciente agora considerado incapaz:

"É um instrumento que só traz benefícios, tanto ao paciente como à familia, porque vai estar respeitando a vontade dele naqueles momentos mais difíceis, de dor, de sofrimento" (EP3).

Os discursos colocam as diretivas como ferramenta facilitadora, já que por meio delas o paciente explicita sua vontade a fim de que sua autonomia seja respeitada ${ }^{17}$. A autonomia é o pilar do processo que se distancia do paternalismo ao ressignificar a relação entre paciente e equipe de saúde, permitindo a atuação efetiva das pessoas no próprio tratamento e possibilitando nova maneira de cuidado ${ }^{24}$. Importantes nesse processo, as DAV permitem ainda liberar a família do fardo de ter que decidir sobre tratamentos que, muitas vezes, não curam a doença, mas recaem na obstinação terapêutica:

"Muitas vezes não sabemos qual é a vontade desse paciente e temos que ficar deduzindo. E até os familiares, é uma questão até emocional de responsabilidade, às vezes, porque eles acham que precisam fazer tudo. Até o familiar, sabendo o que o familiar gostaria de fazer, tiraria um peso na consciência" (EP6).

Também houve falas sobre as DAV no que se refere à necessidade de participação multiprofissional, não apenas para colocá-las em prática, mas também para esclarecer informações que podem constar no documento, de modo a verificar se as vontades manifestadas são de fato exequíveis:

"A gente entra num assunto que eu acredito que não é só da enfermagem; as outras classes têm que explicar muito bem para o paciente os prós e os contras de tratar ou não" (EP2).

"Precisa de uma equipe que saiba o que dizer para o paciente se ele quiser fazer esse documento, porque senão ele pode dizer coisa que não é possivel depois o profissional aceitar" (EP11).

Em seus depoimentos, os enfermeiros ressaltaram que um documento como as DAV apoiaria as ações da equipe no sentido de respeitar a autonomia do paciente mesmo que a família tenha opinião contrária:

"O instrumento dá a possibilidade de o paciente, enquanto lúcido e orientado, se for o caso, optar. Então eu acredito que dá autonomia para essa pessoa dizer o que ela gostaria que fosse feito. Isso dá um respaldo para o profissional, porque muitas vezes é uma vontade do paciente, mas a familia não entende" (EP5).

A maioria dos entrevistados concordou que as DAV ajudariam a harmonizar o compartilhamento de responsabilidades entre profissional de saúde e paciente. A fala dos enfermeiros remete ainda à ideia de que as relações com a família do enfermo podem se tornar conflituosas quando a equipe 
segue as DAV, visto que a expectativa dos familiares pode ser contrária à vontade do paciente. Via de regra, porém, as DAV submetem médicos e familiares à preferência do enfermo, uma vez que este foi informado sobre todas as possibilidades terapêuticas e estava mentalmente lúcido quando registrou suas vontades ${ }^{7,13,20}$.

Nesta categoria foram elencados pontos referentes ao desconhecimento das DAV, à sua importância como instrumento facilitador para a equipe e familiares e à participação multiprofissional. Conclui-se que é necessário ampliar as discussões sobre finitude e questões éticas que a envolvem. Entre as estratégias, destacam-se ações de educação permanente com a equipe de saúde e, em especial, com os enfermeiros, por serem os profissionais mais próximos ao paciente, muitas vezes estabelecendo vínculos fortes. Também é indispensável que essas discussões estejam incluídas na formação de futuros profissionais, preparando-os para situações que poderão vivenciar na prática.

\section{Considerações finais}

Os entrevistados, em geral, aceitam a morte com naturalidade quando ocorre com pacientes portadores de doenças crônicas, e tendem a respeitar, hipoteticamente, o cumprimento das DAV. Alguns enfermeiros, entretanto, ainda desconhecem esse dispositivo e relatam seguir as decisões do paciente apenas se elas não divergirem das rotinas da unidade e da instituição. Após todos os enfermeiros consultarem um modelo de DAV, reconheceram-no como instrumento facilitador para dispor as vontades expressas pelo paciente.

Nas falas, pôde-se identificar desafios que os enfermeiros projetam caso venham a aplicar as
DAV. Dentre eles há o medo de processos judiciais em virtude da ausência de legislação voltada às diretivas antecipadas. Nota-se aqui a importância de que os enfermeiros se sintam seguros para que os avanços da relação profissional-paciente-familiar possam se consolidar. Nesse sentido, é fundamental que toda a equipe multiprofissional conheça as DAV, auxiliando em sua elaboração, esclarecendo informações que podem constar no documento de acordo com a especificidade de cada tratamento e, por fim, colocando o dispositivo em prática.

As DAV oferecem respaldo aos profissionais que as cumprem; o tema, porém, é pouco abordado durante a formação, especialmente na enfermagem. Dessa maneira, é fundamental incluir as DAV no processo formativo, para que os futuros profissionais ajudem seus pacientes a dispor tais diretivas e, depois, possam segui-las sem receio. Da mesma forma, é necessário desenvolver estudos que abordem a temática, visando contribuir para que a morte seja vista com mais clareza e naturalidade pelos profissionais de enfermagem.

As limitações do presente estudo são o cenário da coleta de dados - um único hospital universitário e público - e o fato de os enfermeiros terem sido entrevistados em seu local de trabalho, o que pode ter gerado preocupação e/ou distração, mesmo que não tenham sido demonstradas. A pesquisa revelou demandas relacionadas à aplicação das DAV, instrumento geralmente desconhecido da equipe de saúde. Nesse sentido, o papel do enfermeiro é importante, pois deve ser sensível à sua função junto ao indivíduo provido de DAV, assegurando que toda a equipe preserve os direitos e a autonomia do paciente. O profissional de enfermagem deve perceber deficiências e saná-las sempre que possível, colaborando para a atuação ética ante o paciente em fase final de vida e seus familiares.

\section{Referências}

1. Organização Mundial da Saúde. Resumo: relatório mundial de envelhecimento e saúde. Genebra: OMS; 2015.

2. Viana AP, Reis JM. Distanásia: entre o prolongamento da vida e o direito de morrer dignamente. Rev Bras Direitos Garantias Fundam [Internet]. 2016 [acesso 7 jul 2020];2(2):270-88. DOI: 10.26668/ IndexLawJournals/2526-0111/2016.v2i2.1643

3. Gomes BMM, Salomão LA, Simões AC, Rebouças BO, Dadalto L, Barbosa MT. Diretivas antecipadas de vontade em geriatria. Rev. bioét. (Impr.) [Internet]. 2018 [acesso 7 jul 2020];26(3):429-39. DOI: 10.1590/1983-80422018263263 
4. Arruda LM, Abreu KPA, Santana LBC, Sales MVC. Variables that influence the medical decision regarding advance directives and their impact on end-of-life care. Einstein [Internet]. 2020 [acesso $7 \mathrm{jul}$ 2020];18:eRW4852. DOI: 10.31744/einstein_journal/2020RW4852

5. Dadalto L. A judicialização do testamento vital: análise dos autos n. 1084405-21.2015.8.26.0100/TJSP. Civilistica.com [Internet]. 2018 [acesso 7 jul 2020];7(2):1-16. Disponível: https://bit.ly/3dh1mUg

6. Dadalto L. Living will's history: understanding the past and reflecting about the present. Mirabilia Medicinae [Internet]. 2015 [acesso 7 jul 2020];4:23-42. Disponível: https://bit.ly/3rWPefn

7. Alkimim MA, Rampazzo L. Diretivas antecipadas de vontade (testamento vital): implicações éticas e jurídicas diante do princípio da dignidade da pessoa humana. Rev Bioét Direitos Anim [Internet]. 2016 [acesso 7 jul 2020];2(2):139-58. DOI: 10.26668/IndexLawJournals/2525-9695/2016.v2i2.1380

8. Conselho Federal de Medicina. Resolução CFM n 1.995, de 9 de agosto de 2012. Dispõe sobre as diretivas antecipadas de vontade dos pacientes. Diário Oficial da União [Internet]. Brasília, p. 279-80, 31 ago 2012 [acesso 7 jul 2020]. Seção 1. Disponível: https://bit.ly/3rVPOdd

9. Rodriguez Bermejo I, Villamor Ordozgoiti A, Lahosa Sancho MT, Dorca Corujo M, Más Cagide M, Salvador Fontanet MI. Conocimientos y actitudes de enfermeras de onco-hematología ante el documento de voluntades anticipadas. Rev Cubana Enferm [Internet]. 2018 [acesso 7 jul 2020];34(2):1-13. Disponível: https://bit.ly/2ZgEc8o

10. Nunes MI, Anjos MF. Diretivas antecipadas de vontade: benefícios, obstáculos e limites. Rev. bioét. (Impr.) [Internet]. 2014 [acesso 7 jul 2020];22(2):241-51. DOI: 10.1590/1983-80422014222005

11. Trarieux-Signol S, Bordessoule D, Ceccaldi J, Malak S, Polomeni A, Fargeas JB et al. Advance directives from haematology departments: the patient's freedom of choice and communication with families: a qualitative analysis of 35 written documents. BMC Palliat Care [Internet]. 2018 [acesso 7 jul 2020];17:10. DOI: 10.1186/s12904-017-0265-1

12. Cogo SB, Lunardi VL. Anticipated directives and living will for terminal patients: an integrative review. Rev Bras Enferm [Internet]. 2015 [acesso 7 jul 2020];68(3):464-74. DOI: 10.1590/0034-7167.2015680321i

13. Cassol PB, Quintana AM, Velho MTAC. Diretiva antecipada de vontade: percepção de uma equipe de enfermagem da hemato-oncologia. J Nurs Health [Internet]. 2015 [acesso 7 jul 2020];5(1):4-13. DOI: 10.15210/jonah.v5i1.5497

14. Conselho Federal de Enfermagem. Resolução Cofen $n^{\circ}$ 564, de 6 de novembro de 2017. Aprova o novo Código de Ética dos Profissionais de Enfermagem. Diário Oficial da União [Internet]. Brasília, $n^{\circ} 233$, p. 157, 6 dez 2017 [acesso 7 jul 2020]. Seção 1. Disponível: https://bit.ly/37e9Jfy

15. Saioron I, Ramos FRS, Amadigi FR, Diaz PS. Diretivas antecipadas de vontade: desafios legais e educacionais na visão de enfermeiros. Rev Eletrônica Enferm [Internet]. 2017 [acesso 7 jul 2020];19:a44. DOI: 10.5216/ ree.v19.43587

16. Hospital Universitário de Santa Maria: escalas de plantão. Hospitais Universitários Federais [Internet]. 2020 [acesso 7 jul 2020]. Disponível: https://bit.ly/3jPL4mC

17. Moraes R, Galiazzi MC. Análise textual discursiva. ljuí: Editora Unijuí; 2013.

18. Conselho Nacional de Saúde. Resolução CNS $n^{\circ} 466$, de 12 de dezembro de 2012. Aprova as diretrizes e normas regulamentadoras de pesquisas envolvendo seres humanos. Diário Oficial da União [Internet]. Brasília, $n^{\circ}$ 12, p. 59, 13 jun 2013 [acesso 7 jul 2020]. Seção 1. Disponível: https://bit.ly/3qqd3M2

19. Rocha DD, Nascimento ÊC, Raimundo LP, Damasceno AMB, Bondim HFFB. Sentimentos vivenciados pelos profissionais de enfermagem diante de morte na unidade de terapia intensiva neonatal. Mental [Internet]. 2017 [acesso 7 jul 2020];11(21):546-60. Disponível: https://bit.ly/3ajregp

20. Moreira MADM, Costa SFG, Cunha MLDM, Zaccara AAL, Negro-Dellacqua M, Dutra F. Testamento vital na prática médica: compreensão dos profissionais. Rev. bióet. (Impr.) [Internet]. 2017 [acesso 7 jul 2020];25(1):168-78. DOI: 10.1590/1983-80422017251178

21. Peicius E, Blazeviciene A, Kaminskas R. Are advance directives helpful for good end of life decision making: a cross sectional survey of health professionals. BMC Med Ethics [Internet]. 2017 [acesso 7 jul 2020];18:40. DOI: 10.1186/s12910-017-0197-6 
22. Cogo SB, Lunardi VL, Quintana AM, Girardon-Perlini NMO, Silveira RS. Desafios da implementação das diretivas antecipadas de vontade à prática hospitalar. Rev Bras Enferm [Internet]. 2016 [acesso 7 jul 2020];69(6):1031-8. DOI: 10.1590/0034-7167-2016-0085

23. Salum MEG, Kahl C, Cunha KS, Koerich C, Santos TO, Erdmann AL. Processo de morte e morrer: desafio no cuidado de enfermagem ao paciente e família. Rev Rene [Internet]. 2017 [acesso 7 jul 2020];18(4):528-35. DOI: $10.15253 / 2175-6783.2017000400015$

24. George TP, DeCristofaro C, Murphy PF, Remle CR. Knowledge, attitudes, and experience with advance directives among prelicensure nursing students. J Nurs Educ [Internet]. 2018 [acesso 7 jul 2020];57(1):35-9. DOI: 10.3928/01484834-20180102-07

Silvana Bastos Cogo - Doutora - silvanabastoscogo@gmail.com

(iD) 0000-0002-1686-8459

Elisabeta Albertina Nietsche - Doutora - eanietsche@gmail.com

(D) 0000-0002-8006-2038

Marcio Rossato Badke - Doutor - marciobadke@gmail.com

(D) 0000-0002-9459-1715

Graciela Dutra Sehnem - Doutora - graci_dutra@yahoo.com.br

(D) 0000-0003-4536-824X

Cléton Salbego - Doutorando - cletonsalbego@ hotmail.com

(D) 0000-0003-3734-9970

Tierle Kosloski Ramos - Doutoranda - tierleramos@hotmail.com

(i) 0000-0001-7901-3792

Andrei Pompeu Antunes - Graduando - andrei.pompeuantunes@hotmail.com

(D) 0000-0003-1659-5670

Aline Gomes Ilha - Mestranda - aline.gomes1996@hotmail.com

(D) 0000-0002-5715-9595

Luiza Carolina Santos Malheiros - Graduanda - luizacsmalheiros@gmail.com

(D) 0000-0003-2005-2820

Correspondência

Silvana Bastos Cogo - Av. Roraima, 1.000, Cidade Universitária, Camobi CEP 97105-900.

Santa Maria/RS, Brasil.

Participação dos autores

Os autores contribuíram igualmente para o artigo. 